\title{
IR thermography in heat transfer measurements on a wing leading edge surface internally impinged by a row of air jets
}

by M. Imbriale*, A. Ianiro**, C. Meola*** and G. Cardone ${ }^{\star \star \star \star}$

\begin{tabular}{|c|c|c|c|c|c|c|c|c|}
\hline $\begin{array}{l}{ }^{* *} \text { Dept. of Aerospace } \\
\text { michele.imbriale@unina.it }\end{array}$ & Engineering, & University of & Naples, & via & Claudio & 21 & 80125 & Napo \\
\hline 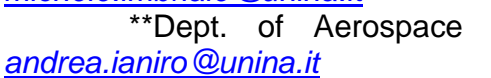 & Engineering, & University & Naples, & via & Claudio & 21 & 80125 & Napoli, \\
\hline $\begin{array}{l}* * \star \text { Dept. of Aerospace } \\
\text { carmeola@unina.it }\end{array}$ & Engineering, & University of & Naples, & via & Claudio & 21 & 80125 & Napoli, \\
\hline $\begin{array}{l}{ }^{* * * \star D e p t . ~ o f ~ A e r o s p a c e ~} \\
\text { gcardone@unina.it }\end{array}$ & Engineering, & University of & Naples, & p.le & Tecchic & $080-$ & 80125 & Napoli, \\
\hline
\end{tabular}

\section{Abstract}

The purpose of this work is the investigation of convective heat transfer through a row of circular air jets internally impinging on an airfoil leading edge surface for anti-icing purposes. Measurements are performed with infrared thermography applied to the heated thin foil technique. Tests are carried out by varying: diameter and mutual spacing of jets, impinging distance, Reynolds and Mach numbers. A geometrical calibration of the infrared camera is performed. Data is presented in terms of Nusselt number maps. A new data correlation is proposed between the average Nusselt number and the Reynolds number, by accounting for the impingement angle.

\section{Introduction}

The ice formation on wing and engine inlets leading edges can dramatically compromise aircraft performance and operation. Then, the ice formation and accretion is receiving great interest [1] and several methods of ice prevention and removal have been designed. In particular, ice formation on wings and engine inlets of modern commercial transport aircraft can be prevented by extracting hot air from the compressor and blowing it on the inside surface of the leading edge through small holes drilled in a pipe [2] in order to supply enough energy to evaporate the impinging water or to maintain the surface at a temperature above the freezing value.

The high heat transfer rate that is possible to obtain with impinging jets is well recognized and explained in a wide scientific literature [3] with also correlations for heat and mass transfer [4].

The first work concerned with impinging jets as anti-icing device is perhaps that of Jusionis [5] who addressed the case of jets impinging inside a closed surface, which simulated a wing profile. He performed heat transfer measurements using thermocouples attached to the target surface and proposed as characteristic length in Nusselt and Reynolds numbers an effective slot width $S^{*}$ including hole diameter and hole-to-hole spacing.

Several papers concerned with impinging jets on concave surfaces are available [6-7]. Bunker and Metzger [6] find out a general increase in heat transfer with decreasing the leading edge sharpness as well as with decreasing the nozzle to apex distance, and increase in span-wise average heat transfer with decreasing the jet pitch to diameter ratio. Fenot et al. [7] study the heat transfer due to a row of hot jets impinging on a concave surface and present correlations of effectiveness and Nusselt number at stagnation points. They find that the confinement effect prevents cold ambient air from mixing with hot air jet and thereby reducing the flow temperature. In particular, the fountain effect, due to the mixing of neighbouring jets, also prevents jet cooling. The relative curvature is found to have two opposite effects on the Nusselt number distribution. The first involves an overall reduction of the Nusselt number. The second effect, instead, is an enhancement of heat transfer near the impinging zone, which is probably due to the increase in relative curvature.

A main problem with a jet impinging on a curved surface is certainly the impingement angle. In fact, in some applications, due to geometrical constraints, or jets mutual interference, the impingement may be oblique. It is also worth noting that the jet, even if normally directed, impinges normally only in the stagnation point, while it spreads out nearby under a certain angle; the area surrounding the stagnation point is of great concern in most cases. In this context, is worth attention the work by Tawfek [8] who was the first to address the effects of oblique impingement upon a curved (convex) surface. He observed an increasing asymmetry around the point of maximum heat transfer with the impinging angle, which had no great effects on the average heating/cooling jet performance. Later, Lee et al. [9] studied the effects of the angle impingement on the heat transfer over a concave surface using the liquid crystal transient method. They observed the maximum Nusselt number to occur in the upstream direction with respect to the stagnation point and an increased displacement of such a maximum with increasing the surface angle or decreasing the nozzle-to-surface distance.

In this work the heat transfer performance of rows of circular jets internally impinging on the airfoil leading edge region is experimentally studied by means of infrared thermography with the heated thin foil heat transfer sensor [10]; the 
Nusselt number distribution is shown in 3D rebuilding [11]. Furthermore, a new data correlation between the average Nusselt number and the Reynolds number, by accounting for the impingement angle, is proposed.

\section{Experimental Test.}

The investigation is carried out with infrared thermography (IRT) applied to the heated thin foil technique [10]; this has required a specific setup and test procedure as will be described in the following sections 2.1 and 2.2. It is worth noting that the experimental apparatus is similar to that used by Meola et al. [12], but with the substantial novelty that the temperature maps are rebuilt on the object mesh grid according to Cardone et al. [11]. This involves not only a different data reduction, but primarily a geometrical calibration of the infrared camera as will be described in section 2.3.

\subsection{Test Setup}

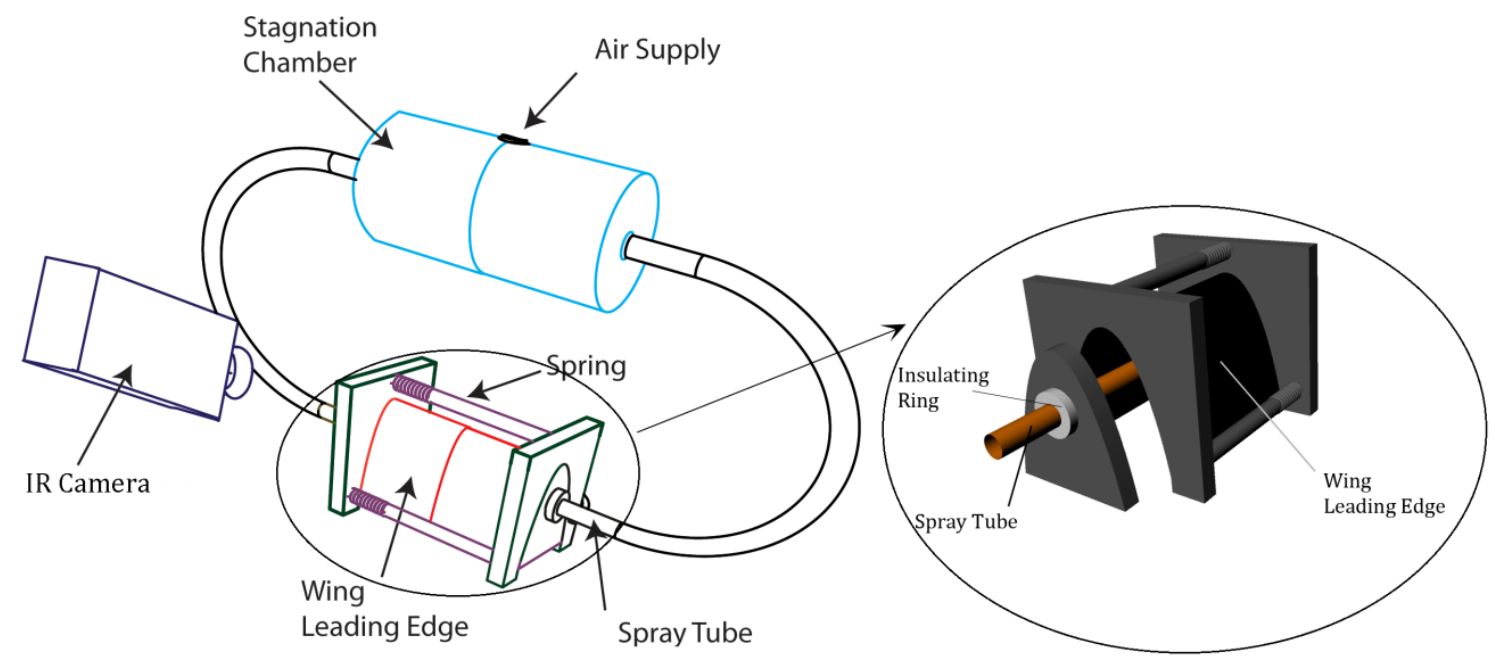

Fig. 1 Experimental apparatus.

The main experimental apparatus component is the leading edge section of a NACA 0012 profile with chord $c=$ $1.50 \mathrm{~m}$ reproduced by a stainless steel foil ( $40 \mu \mathrm{m}$ thick) heated by electric current passing through it and cooled by the jets impinging on it. Two moulding stainless plates allow the thin foil to reproduce the NACA profile and at the same time they operate such as electric contacts (Fig. 1). To avoid any foil thermal deformation and waving, the moulding plates are equipped with two settable springs that convey a positive span-wise strength to the foil. To reduce measurement errors due to reflections, the foil is coated with a thin film of high emissivity paint $(\varepsilon=0.95)$.

The wing profile or test profile is $0.200 \mathrm{~m}$ long span-wise and it is stopped about $1 / 10$ of the chord because this is the most critical zone for icing occurrence. Two replaceable flanges, made of insulating material allow positioning of the spray tube (external diameter equal to $25 \mathrm{~mm}$ and thickness equal to $2 \mathrm{~mm}$ ) inside the wing cavity.

The spray tube has a certain number of aligned holes to reproduce periodic effects of jets impinging. Rotating the spray tube around its axis it is possible to assess the influence of the jets angle on the heat transfer coefficient distribution. It is fed from both sides by air coming from the stagnation chamber, which (Fig.1) is positioned on the top. In more details, the air flow, supplied by a high pressure tank (up to 60 bar), goes through a pressure regulator valve, then to the stagnation chamber where it splits into two equal quantities to fed the spray tube from both extremities and guarantee uniform distribution of air through the tube length. Finally, air is spread out by the holes. In particular, different spray tubes are used with the number of holes varied from 3 to 5, the hole diameter $d$ varied from $2 \mathrm{~mm}$ to $4 \mathrm{~mm}$, and pitch on diameter ratio (the holes spacing $p$ ) varied between 5 and 15 . In addition, it is also varied the jet angle $\phi$ from $0^{\circ}$ to $50^{\circ}$ by rotating the spray tube around its axis. The Mach number is varied from 0.6 to 1.0 .

The used infrared system is based on a CEDIP Jade IR camera with a FPA of $320 \times 240$ pixels detector working in the $3.8-4.5 \mu \mathrm{m}$ infrared band. Nominal sensitivity, expressed in terms of Noise Equivalent Temperature Difference, is $20 \mathrm{mK}$ at $30^{\circ} \mathrm{C}$

\subsection{Test Procedure}

As already said, heat transfer measurements are performed owing to the heated thin foil sensor for which the convective heat transfer coefficient $\mathrm{h}$ is given by the following relationship:

$$
h=\frac{\dot{q_{J}}-\dot{q_{r}}-\dot{q_{k}}-\dot{q_{n}}}{\left(T_{w}-T_{a w}\right)}
$$


where $\dot{q}_{j}$ is the Joule heating per unit area, $\dot{q}_{r}$ is thermal radiation heat flux, $\dot{q}_{n}$ is natural convection heat flux and $\dot{q}_{k}$ is the foil lateral thermal conduction heat flux. $T_{w}$ and $T_{a w}$ are wall and adiabatic wall temperatures that are measured on the foil surface opposite to jets impingement (see Fig.1); (i.e., jets impinge internally on the leading edge while the infrared camera views the external surface). This is possible since the Biot number lowness $\left(B i=h t / k_{\text {steel }} \cong 10^{-4}, t\right.$ being the foil thickness) for which the temperature can be considered instantaneously the same on both the foil sides.

By assuming the ambient as a black body at a constant temperature and the sensor surface gray in the wavelength band of interest (assumptions that can be made in this case), the net rate of radiation heat loss can be estimated as:

$$
\dot{q}_{r}=\varepsilon \sigma\left(T_{w}^{4}-T_{a m b}^{4}\right)
$$

Thermal conduction heat flux is evaluated through a laplacian operator of temperature following Astarita and Cardone [13] and it is found to be less than $3 \%$ of the heat flux provided by Joule effect.

The natural convection heat flux $\dot{q}_{n}$ can be neglected because, in the worst experimental condition (maximum temperature difference between foil and air and minimum exit Mach number), it is:

$$
\frac{G r}{\operatorname{Re}^{2}}=\frac{\bar{g} \beta\left(T_{w}-T_{a m b}\right) L}{w^{2}}<<1
$$

where $G r=\bar{g} \beta\left(T_{w}-T_{a m b}\right) L^{3} / v^{2}$ is the Grashof number based on the characteristic length $L$ equal to $10 \%$ of chord and $R e=w L / v$ is the Reynolds number. To measure both wall and adiabatic wall temperatures (i.e. $T_{w}$ and $T_{\text {aw }}$ ) each test run is composed of two parts. First, without electric heating, with the jets impinging and once steady state conditions are reached, a thermal image (or a sequence) is recorded that is called cold image and provides $T_{\text {aw }}$ values; then, the electric current is put on and the so-called hot image is recorded which provides $T_{a w}$ values. Indeed, both $T_{a w}$ and $T_{w}$ are obtained by averaging 500 thermal images acquired in time sequence at $10 \mathrm{~Hz}$; this helps filtering data from natural fluctuations phenomena. Of course, this procedure is repeated for all the test conditions which include change of the spray tube (i.e., variation of holes diameter and spacing), jet angle as well Reynolds and Mach numbers. Particular attention deserves the jet impingement inclination with respect to the chord-wise direction. This parameter may be confused with the impingement distance; however, because of the chord-wise surface curvature variation, it is more appropriate to refer to the jet angle $\phi$ rather than to the impingement distance.

The acquired thermal images are first analyzed in terms of temperature maps of $T_{a w}$ and $T_{w}$ and then reduced in dimensionless form in terms of the Nusselt number $\mathrm{Nu}$ :

$$
N u=\frac{h d}{k}
$$

where $h$ is the convective heat transfer coefficient defined in Eq. (1), $d$ is the hole diameter and $k$ is the air thermal conductivity evaluated at film temperature. It is evaluated also the Nusselt number mean distribution:

$$
N u_{\text {mean }}=\frac{1}{2 p} \int_{y^{*}-p}^{y^{*}+p} N u(x, y) d y
$$

where $y^{*}$ is the spanwise coordinate of the central jet centre and $x$ is the chordwise coordinate.

\subsection{D Rebuilding}

Before Nusselt number calculation the temperature maps have to be rebuilt on a 3D mesh grid from 2D IR images and the first step involves geometrical camera calibration according to pin-hole camera model with an ad-hoc target. More specifically, a special support with five target tracks, called calibration target, has been designed in order to calibrate the volume occupied by the airfoil leading edge (see a scheme of the setup in Fig. 2).

For every test, the calibration target is recorded in 6 positions at distance of $5 \mathrm{~mm}$ between them sweeping the volume of temperature measurements.

The target is made of aluminum with regularly spaced dots on a matrix of 9x12 (see details in Fig. 2). The dots are obtained with high emissivity paint and the target is heated from its backside.

Once, both the real-world coordinates of these markers and the markers positions in the image plane are known, the parameters of the camera model (that allow projection of the real-world markers in their corresponding image locations) are determined. The temperature maps are finally rebuilt on the grid (Fig. 3) representative of the observed surface by means of the camera model. In fact, the back-projection of all the points of the surface grid, with the camera model, allows obtaining, by interpolation, the measured Nusselt number values corresponding to every points. Of course, the mesh grid reproduces the NACA 0012 leading-edge region with the same spatial resolution of the IR images in order to avoid drop of spatial resolution and increase of computational costs. 


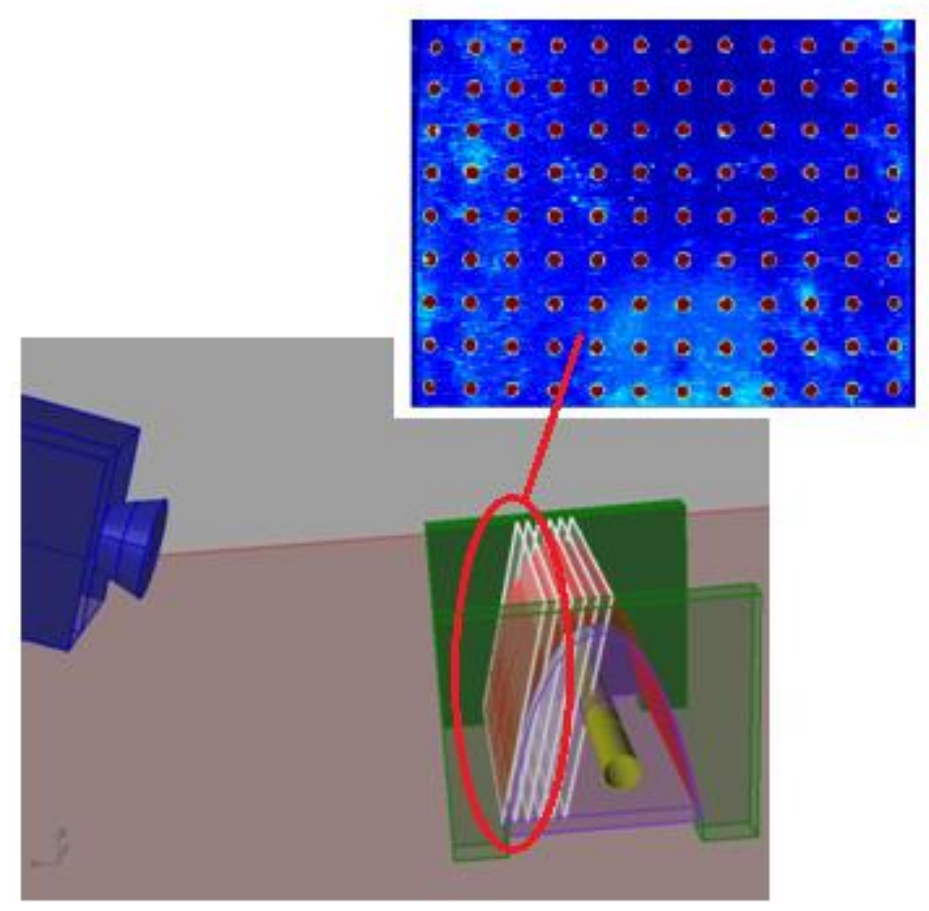

Fig. 2 Test Target. In the present picture the test target (white surface) and the profile (red surface) are shown together to better link the meaning of measurement volume. They are severally mounted between clamps.

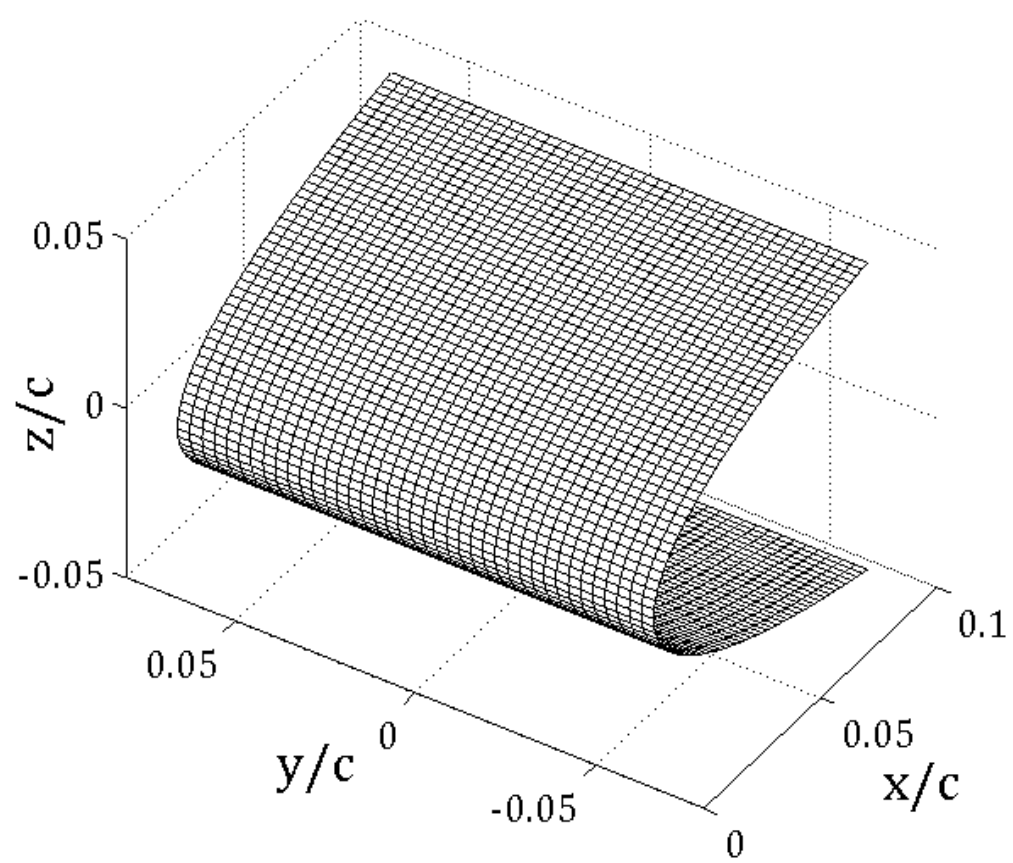

Fig. 3 3D mesh grid of the NACA-0012 leading edge.

To obtain the temperature distribution over the entire leading edge surface, at least two images must be acquired; one taken on the front-side with jets impinging on the rear (i.e., the side opposite to jets impingement) and the other one taken on the backside without any jets impingement. This obviously, entails a change of position of the infrared camera, or a $180^{\circ}$ rotation of the leading edge profile that inevitably involves rotation of the entire test apparatus. Because of the profile symmetry, the acquisition of the two images can be simplified by taking both on the same surface by simply rotating the spray tube to comply with both of the conditions with and without jets impingement. Afterwards, the two images have to be rebuilt together on a 3D mesh grid. An example of the so obtained images is given in figure 4 for Tw (Fig.4a) and Taw (Fig.4b). 
(a)

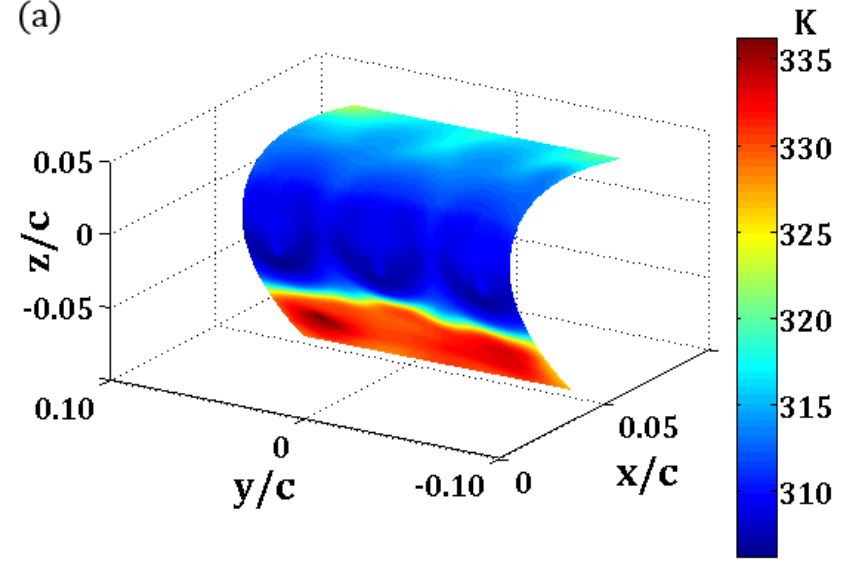

(b)

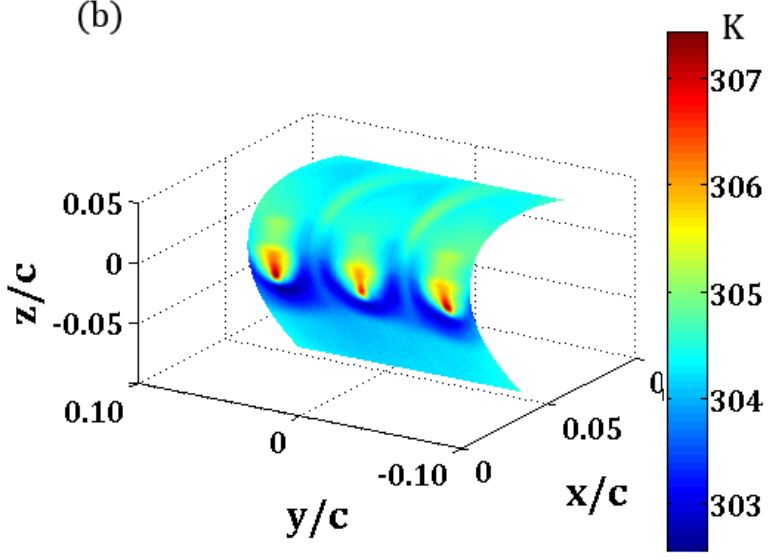

Fig. $43 D$ Temperature rebuilding for $d=4 \mathrm{~mm}, \phi=20^{\circ}, M=1.0$ and $p / d=10$. (a) shows the wall temperature (with foil heating) while (b) shows the adiabatic wall temperature (without foil heating).

In figure 5 is shown a typical example of the Nusselt number map rebuilt on the leading edge surface. The jets, impinging on leading edge, provide very high Nusselt number values in a small region with localized peaks corresponding to jet impingement. So, the Nusselt number peaks clearly locate the area of jet impingement on the frontside surface of 3D rebuilding.

Even though the holes are perfectly circular, the high Nusselt region has tendency to stretch in chordwise direction. This behaviour is due to the jets inclination with respect to the foil surface.

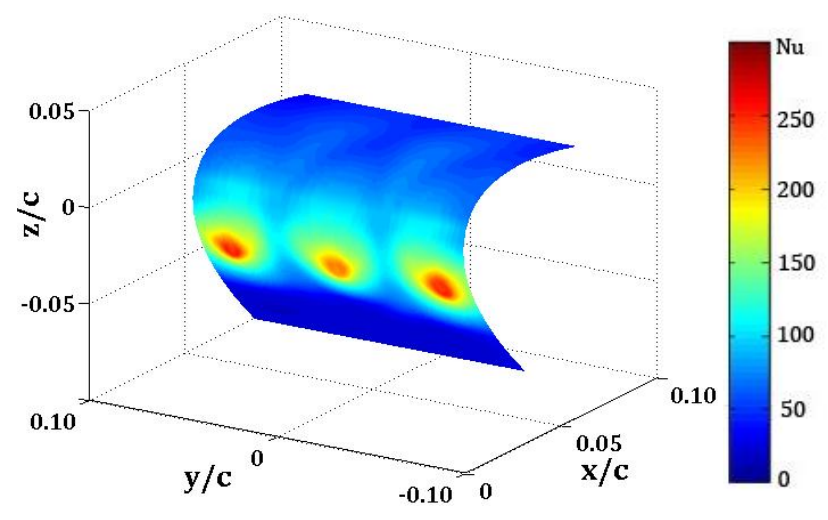

Fig. 5 Nusselt Map on a $3 D$ mesh grid for $M=1.0, p / d=10$ and $\phi=20^{\circ}$.

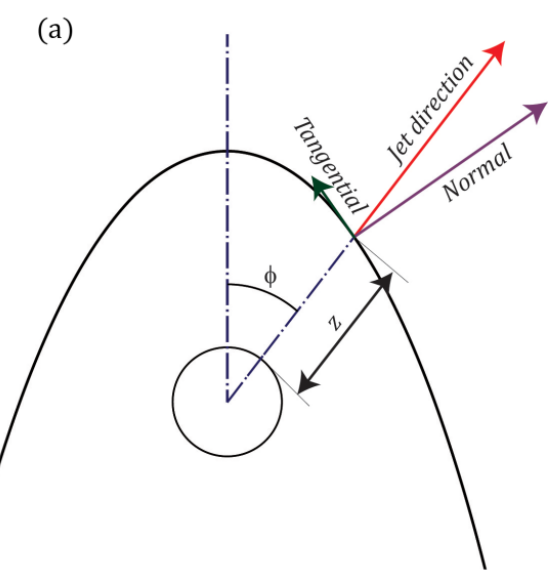

(b)

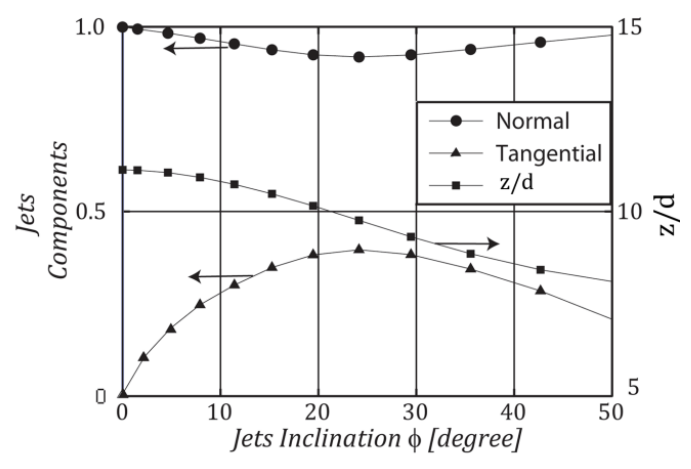

Fig. 6 Projection over Leading Edge Surface in dependence of jets angle Inclination.(a) shows the jets components in reference with the leading edge, (b) shows the dimensionless component of jets velocity (left axes) and the dimensionless nozzle to surface distance (right axes). 


\section{Data Analysis}

An important parameter is the jets inclination $\phi$ being the target surface curved; of course, $\phi$ is closely linked to the nozzle-to-surface distance $z$ as shown in figure 6 . In particular, $z$ made dimensionless with respect to the nozzle diameter $d$ is plotted against $\phi$ with also normal and tangential components in figure $6 \mathrm{~b}$. As can be seen, $z / d$ decreases with increasing the jets angle.

The spray tube is positioned at $4 \%$ of the chord profile; in this configuration, the nozzle to surface distance increases as the jets angle decreases. Hence, the peaks of Nusselt number strengthen as the jets inclination increases from $0^{\circ}$ to $50^{\circ}$ (Fig.7a-e). This trend is better demonstrated through the plotted Nusselt mean distributions in figure $7 \mathrm{f}$ for different values of jets angle. In particular, for $\phi=50^{\circ}$ (corresponding to the minimum value of $z / d$ ) the peak value attains its maximum.

It has to be noted that the Nusselt distributions shown in figure 7 refer to $p / d=10$ and $M=1$. Of course, the peaks are recorded at chordwise coordinate corresponding to the row jets impingement.

(a)

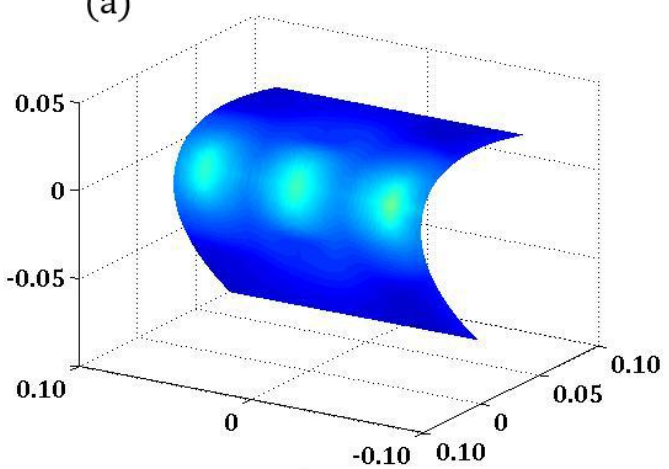

(c)

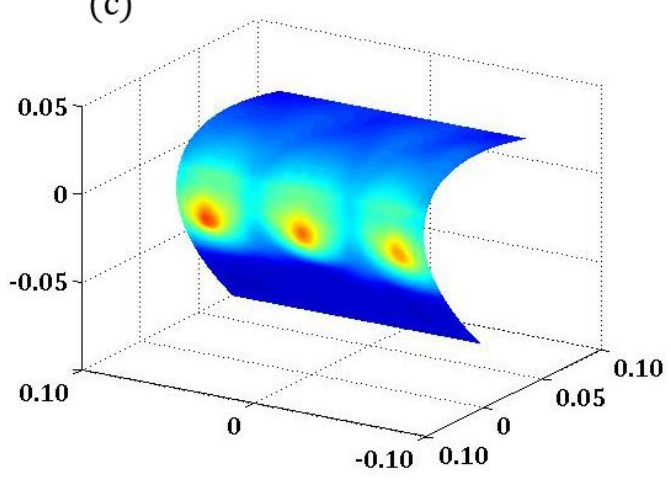

(e)

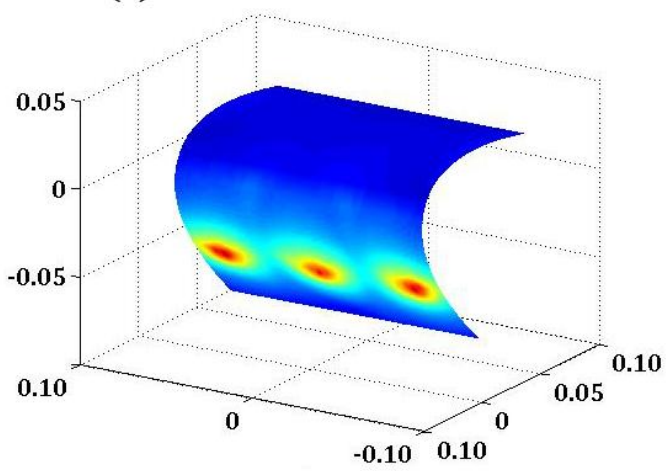

(b)

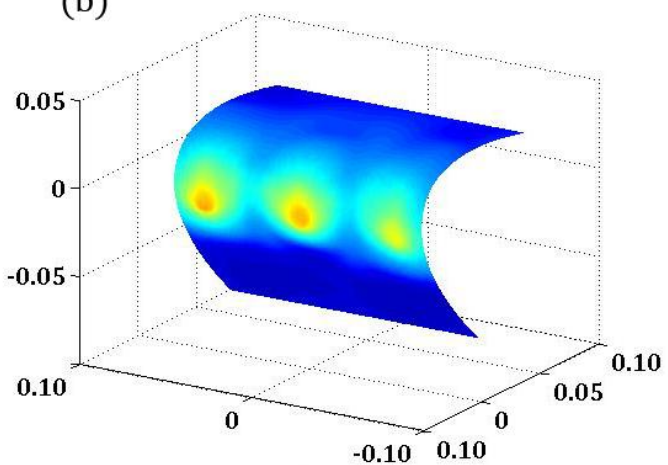

(d)

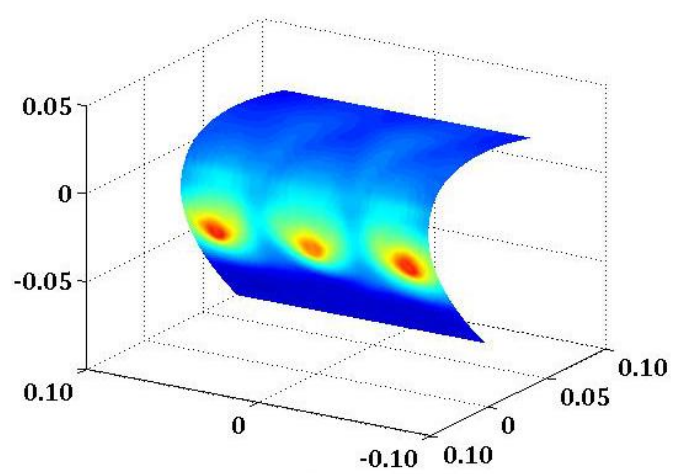

(f)

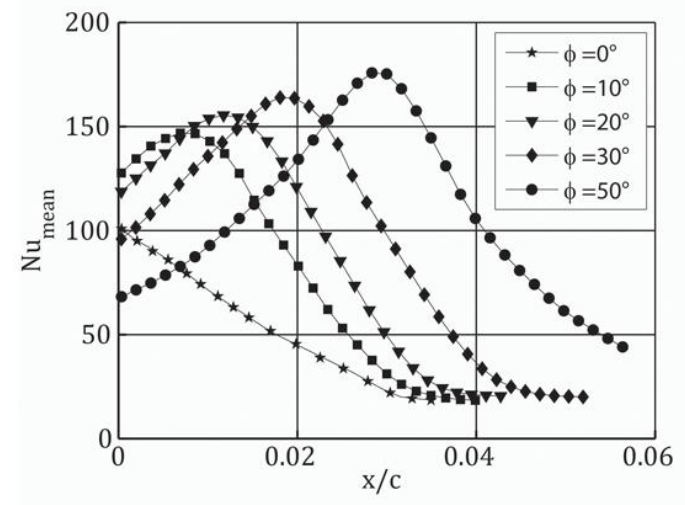

$\mathrm{Nu}$

250

200

150

Fig. 7 Jet angle dependence on Nusselt maps distribution $p / d=10$ and $M=1.0$. 


\subsection{Data Correlation}

Heat transfer correlations are obtained with reference to the $N u$ values over the impingement area $A$, which is the surface obtained by the intersection between the surface of the wing profile and a cylinder that has the same axes of the jet and a radius equal to the hole-to-hole spacing. Both Reynolds and Nusselt numbers are modified to take into account the hole to hole spacing $p$. Then, they are based on the equivalent slot length $s$ proposed by Jusionis [5]:

$$
s=\frac{\pi d^{2}}{4 p}
$$

In this region the modified Nusselt number is averaged:

$$
\overline{N u_{s}}=\frac{1}{A} \int_{A} N u_{s} d A
$$

then a correlation with the modified Reynolds number is carried out. It is worth noting that Jusionis [5] found a data correlation of the type:

$$
\overline{N u_{s}}=\boldsymbol{a}\left(\frac{z}{s}\right)^{\boldsymbol{b}} R e_{s}^{f}
$$

where $\mathbf{a}=\mathbf{0 . 0 3 0}, \boldsymbol{b}=-\mathbf{0 . 4}$ and $\boldsymbol{f}=\mathbf{0 . 7}$.

In this work, a new kind of data correlation is proposed, which accounts also for the impingement angle $\phi$ :

$$
\overline{N u_{s}}=\boldsymbol{a}\left(\frac{z}{s}\right)^{\boldsymbol{b}} \operatorname{Re}_{s}{ }^{f} \tan (\phi)^{g}
$$

where $\mathbf{a}=\mathbf{0 . 2 7}, \mathbf{b}=\mathbf{- 0 . 6 7}, \mathbf{f}=\mathbf{0 . 7 5}$ and $\mathbf{g}=\mathbf{- 0 . 1 4}$ with a correlation coefficient $\boldsymbol{r}^{2}$ equal to $\mathbf{0 . 9 9 1 2}$. The $\overline{\mathbf{N u}} \bar{s}$ values are plotted against $\boldsymbol{R e}_{\boldsymbol{s}}$ in figure 8 together with eq.(9).

It has to be outlined that the range of applicability of the Jusionis' relationship is: $1000<\operatorname{Re}_{\mathrm{s}}<8000,50<$ $z / s<120$, and $d=2.5 \mathrm{~mm}$, while the present experimental data refer to two different diameters of the holes of the spray-tube $d=2 \mathrm{~mm}$ and $d=4 \mathrm{~mm}, \boldsymbol{z} / \boldsymbol{s}$ varying from 35 up to 354 and $\boldsymbol{R} \boldsymbol{e}_{\boldsymbol{s}}$ in the range $1600-20000$. Thus, the proposed correlation embraces a broader range of variation of the involved parameter; however, the main novelty lies in the impingement angle, which is firstly accounted for.

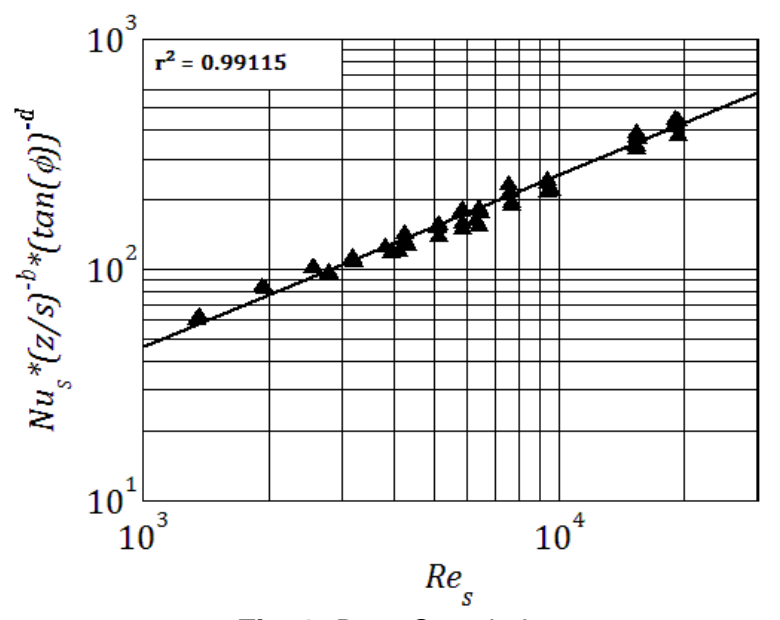

Fig. 8. Data Correlation

\section{Conclusions}

An experimental investigation was carried out on heat transfer by a row of jets impinging internally on a NACA 0012 wing leading edge for anti-icing purposes. Measurements were performed with infrared thermography and the heated thin foil heat transfer sensor. The acquired thermal images were rebuilt in the 3D surface of the wing leading edge through previous geometrical calibration of the infrared camera. The analysis of 3D images allows for a better insight in the main features of the heat transfer distribution. As a main result, heat transfer measurements in the impingement region are collected in a novel correlation that relates the Nusselt number with the other main parameters such as Reynolds number, nozzle to plate distance and impingement angle. 


\section{REFERENCES}

[1]. T. Cebeci, F. Kafyeke, Aircraft Icing, Annu. Rev. Fluid Mech. 2003. 35:11-21.

[2]. W. B. Wright An Evaluation of Jet Impingement Heat Transfer Correlations for Piccolo Tube Application, NASA/CR-2004-212917.

[3]. H. Martin, Heat and Mass Transfer between Impinging Gas Jets and Solid Surfaces, Advances in Heat Transfer, vol. 13, 1-60, 1977.

[4]. C. Meola, A New Correlation of Nusselt Number for Impinging Jets, Heat Transfer Engineering, vol. 30:3, 221-228, 2009.

[5]. Jusionis, V. J. Heat transfer from impinging gas jets on an enclosed concave surface, J. Aircraft, vol. 7,1, 87-88, 1970.

[6]. R.S. Bunker, D.E. Metzeger, Local Heat Tranfer in Internally Cooled Turbine Airfoil Leading Edge Regions: Part I Impingement Cooling Film without Coolant Extraction, Journal of Turbomachinery, Vol.112/451, 1990.

[7]. M. Fenot, E. Dorignac, J.-J. Vullierme, An experimental study on hot round jets impinging a concave surface, International Journal of Heat and Fluid Flow vol. 29, 945-956, 2008.

[8]. Tawfek, A.A., Heat Transfer Studies of the Oblique Impingement of Round Jets upon a Curved Surface, Heat and Mass Trans., vol. 38, 467-75, 2002.

[9]. C.H. Lee, K.B. Lim, S.H. Lee, Y.J. Yoon, N.W. Sung. A study of the heat transfer characteristics of turbulent round jet impinging on an inclined concavesurface using liquid crystal transient method, Experimental Thermal and Fluid Science, vol. 31, 559-565, 2007.

[10].G. Cardone, A. Ianiro, G. Dello loio, A. Passaro, Temperature Maps Measurements on 3D Surface with Infrared Thermography, Experiments in Fluids, vol. 52, 375-385, 2012.

[11].G.M. Carlomagno, G. Cardone, Infrared Thermography for Convective Heat Transfer Measurements, Experiments in Fluids, vol. 49, 1187-1218, 2010.

[12].Meola, C., Carlomagno, G. M., Riegel, E., Salvato, F., An experimental study of an anti-icing hot air spraytube system. 19th Congr. Int. Council Aeronaut. Sci., ICAS-94-2.7.1, Vol. 3, pp. 2345-2351, Anaheim, 1994.

[13]. Astarita T., Cardone G., Thermofluidynamic analysis of the flow in a sharp $180^{\circ}$ turn channel, Experimental Thermal and Fluid Science, vol. 20, 188-200, 2000. 